
\title{
28 Research Square \\ Vitamin D3 controls TLR4- and TLR2-mediated inflammatory responses of endometrial cells
}

\section{Alireza Ghanavatinejad}

Pasteur Institute of Iran

\section{Nesa Rashidi}

Immunology Research Center (IRC), Iran University of Medical Sciences, Tehran, Iran

\section{Mahroo Mirahmadian}

Department of Immunology, School of Medicine, Tehran University of Medical Sciences, Tehran, Iran

\section{Simin Rezania}

Biophysics Institute, Medical University, Austria of Graz,

\section{Mahdokht Mosalaei}

Reproductive Immunology Research Center, Avicenna Research Institute, ACECR, Tehran, Iran Jamileh Ghasemi

Reproductive Immunology Research Center, Avicenna Research Institute, ACECR, Tehran, Iran Amir-Hassan Zarnani ( $\nabla$ zarnania@tums.ac.ir)

Tehran University of Medical Sciences

\section{Research article}

Keywords: Vitamin D3, Endometrium, Inflammation, Toll like receptors, Pro-inflammatory cytokines

Posted Date: December 23rd, 2019

DOI: https://doi.org/10.21203/rs.2.19548/v1

License: (c) (i) This work is licensed under a Creative Commons Attribution 4.0 International License. Read Full License

Version of Record: A version of this preprint was published at Gynecologic and Obstetric Investigation on January 1st, 2021. See the published version at https://doi.org/10.1159/000513590. 


\section{Abstract}

Background: There is a significant association between intrauterine infection-associated inflammatory responses and such pregnancy complications as abortion and preterm labor. Here, we aimed to investigate anti-inflammatory effects of 1,25 $(\mathrm{OH}) 2$ D3 on pro-inflammatory cytokines secretion and expression of TLR2, TLR4 and MyD88 in endometrial stromal cells (ESCs) and whole endometrial cells (WECs).

Method: WECs were treated with either lipopolysaccharide (LPS) or lipoteichoic acid (LTA) and ESCs were treated with LPS. IL-6, IL8 and TNF-a were quantified using ELISA technique. TLR2, TLR4 and MyD88 expression were assessed by RT-qPCR. TLR4 expression at the protein level was studied by Western blot technique.

Results: 1,25 (OH)2 D3 significantly reduced TNF-a production in LPS-activated ESCs and TNF- $a$ and IL-6 production by LTA-stimulated WECs. In contrast, 1,25 $(\mathrm{OH}) 2$ D3 pre-treatment increased production of IL-8 by LPS- and LTA-stimulated endometrial cells. 1,25 $(\mathrm{OH}) 2$ D3 pre-treatment markedly reduced LPSinduced TLR-4 protein expression by ESCs. LPS treatment of ESCs significantly induced MyD88 gene expression. This effect was reversed when these cells were pre-treated with 1,25 (OH)2 D3 before stimulation with LPS.

Conclusion: 1,25 $(\mathrm{OH}) 2$ D3 is an immunomodulatory molecule essential for maintenance of endometrial immune homeostasis through controlling potentially harmful inflammatory responses associated with female reproductive tract infections. Key words: Vitamin D3, Endometrium, Inflammation, Toll like receptors, Pro-inflammatory cytokines

\section{Background:}

Intrauterine infections during pregnancy are among the leading cause of pregnancy-associated complications such preterm labor, preeclampsia and abortion [1]. Endometrium, where implantation occurs, should govern a finely-tuned and controlled immune responses against developing embryo and at the same time prevent ascending infections which could be potentially harmful to gestation. This tissue is influenced by pathogens invading vagina and cervix resulting to endometrial infection and pregnancy failure complications. Thus, to accomplish a normal pregnancy, there should be intrinsic mechanisms controlling infections in the endometrium [2].

Toll like receptors (TLRs) are the main trigger of innate immune responses [3]. Up to now, ten TLR members have been identified in human and each member recognizes specific pathogen-associated molecular patterns (PAMPs). TLRs play an important role in immune defense against pathogens in such mucosa bearing organs as respiratory and digestive tracts [4]. Data collected during the past decade also highlighted their eminent role in immunity of reproductive tract. A plenty of reports have shown the expression of TLR1-6 and 9 in human endometrium $[5,6]$. At the protein level, immunohistochemical experiments have also confirmed presence of TLR1-6 at different areas of female reproductive tract (FRT) 
[7]. TLRs signal through the recruitment of specific adaptor molecules. Binding of TLR2-4 to PAMPs recruit myeloid differentiation primary- response protein 88 (MyD88), the key protein adapter in signal transduction of TLRs, leading to activation of JNK, NF-KB and P38 MAP-kinase and expression of proinflammtory cytokines such as TNF- $a$, IL-6 and IL-1 [8].

While pro-inflammatory cytokine responses are essential for the early and late events of pregnancy, an uncontrolled inflammatory response that may occur during an unresolved infection, could potentially lead to unfavorable pregnancy outcome. Binding of lipopoysaccharide (LPS) to TLR4 induces production of pro-inflammatory cytokines from trophoblasts and binding of lipoteichoic acid (LTA) to TLR2 reduces longevity of these cells and induce their apoptosis which has negative effects on placental growth and gestation [9]. There are evidence showing association of pro-inflamatory cytokines and poor ourcom pf pregnany. Injection of TNF-a, IFN-y and IL-2 to pregnant mice inhibits trophoblasts growth and leads to abortion [10]. Some clinical studies also indicated that serum levels of pro-inflammatory cytokines in women with history of spontanuous abortion are elevated compared with normal controls [11]. Indeed, significant amount of pro-inflammatory cytokines such as IL-1, TNF-a, IL-6 and IL-8 present in aminotic fluid of women who have experienced preterm birth [12]. Therfore, therapeutic modalitis for controling infection-associated inflammatory responses during pregnancy would be desirable.

1,25 dihydroxycholecalciferol $(1,25(\mathrm{OH}) 2 \mathrm{D} 3)$ is the active form of vitamin D3 which beside regulating clacium and bone metabolism, has potent immunomodulatory features [13]. Biological effects of 1,25 $(\mathrm{OH}) 2 \mathrm{D} 3$ are exerted through its receptor; vitamin D receptor (VDR) which is a member of nuclear superfamily receptors and is expressed in many tissues including male and female reproductive tract [14-16]. 1,25 (OH)2 D3 modulates pro-inflammatory cytokines production through interfering with nuclear transcription factors such as NF-AT and NF-KB or via direct interaction with vitamin D3 response element in the promotor region of those cytokines which compromises transcription of key Th1 cytokines such as; IL2 and IFN-Y and shifts immune response towards a Th2 profile. 1,25 (OH)2 D3 also conducts its anti-inflammatory action by reducing $\mathrm{MHCll}$ and co-stimulatory molecules expression on and inhibition of IL-12 production by antigen presenting cells preventing T cell activation by a DC-dependent mechanism $[17,18]$. There are few studies addressing immunomodulatory effects of 1,25 (OH)2 D3 on TLRs expression in monocytes and macrophages [19], but threre is no evidence on potential immunomodulatory activity of 1,25 (OH)2 D3 on TLR-mediated endometrial inflammatory responses. Here we hypothesized that 1,25 $(\mathrm{OH}) 2 \mathrm{D} 3$ could amliorate inflammatory resposes associted with stimulation of human endometrial cells with TLR2 and TLR4 ligands.

\section{Methods And Materials:}

Subjects:

Ethical committee of Avicenna Research Institute approved all experiments in this study and samples were collected after getting signed consent forms from participants. Endometrial tissues were obtained from fifteen apparently normal women between $25-45$ years of age referring to Avicenna infertility clinic 
for hysteroscopic tubal ligation who had no evidence of pathology in their hysteroscopic evaluations. Participants had no history of infertility, miscarriage or preeclampsia, thyroid disease, sign of vaginal infection or confounding pathology such as myoma in their endometrium and all had history of at least one successful delivery. All subjects had regular menstrual cycles and did not take vitamin D3 or hormone in the last three months.

Sample collection:

Endometrial samples were collected by gynecologists using pipelle pipette in proliferative phase of menstrual cycle and transferred to the laboratory in a cold chain in DMEM containing antibiotics in the sterile condition. Samples were divided into three parts for pathological examination, RNA extraction, and cell isolation. Sample for RNA extraction was immediately immersed in RNAlater and frozen at -20区 C.

Isolation of whole endometrial cells (WECs) and stromal cells (ESCs)

Isolation of WECs and ECSs were preformed according to the protocol published elsewhere [20]. In brief, tissues pieces were cut into small pieces and digested for $1.5 \mathrm{~h}$ at $37 \mathrm{C}$ with collagenase $\mathrm{D}$ and DNAse (Roche, USA). Isolated cells were used as WECs. For isolation of ESCs, a part of WECs was cultured for $24 \mathrm{~h}$ and adherent cells were collected. Purity of isolated ESCs were determined by immunofluorescent staining and flow cytometry. Vimentin+, nestin+, cytokeratin-, CD10 + and CD45- cells were regarded as ESCs [20].

Treatment with LPS, LTA and 1,25 (OH)2 D3

We previously showed that WECs expressed TLR2 and TLR4, while ESCs only expressed TLR4 [20] and therefore; in this study WECs were treated with either LPS or LTA, while ESCs were exposed to only LPS. Indeed, using a kinetic study on production of pro-inflammatory cytokines following LPS and LTA treatment of WECs and ESCs, we had previously shown that LTA with the concentration of $1000 \mathrm{ng} / \mathrm{ml}$ were optimal doses to drive pro-inflammatory cytokines by those cell types [20]. To this end, WECs and ESCs were treated with pre-optimized concentration of LTA or with 100-10000 ng/ml LPS. Moreover, according to our previous reports[15, 16, 21], physiologic concentration of vitamin D3 was selected for assessing its effects on LPS- and LTA-induced target genes and proteins expression. To evaluate the effects of 1,25 $(\mathrm{OH}) 2$ D3 on cytokines production by WECs and ESCs, $1 \times 10^{5}$ cells were cultured in wells

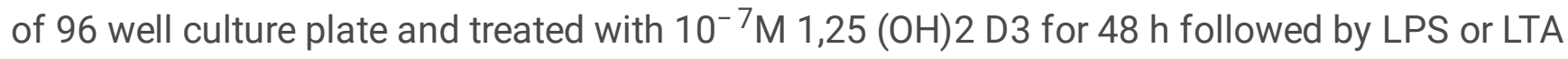
stimulation for $24 \mathrm{~h}$. Conditioned media were then harvested and stored at $-70 \mathrm{C}$ cytokine assay. In order to assess potential effect of 1,25 (OH)2 D3 on modulation of LPS- or LTA-stimulated expression of TLR2, TLR4 and MyD88, $10^{6}$ endometrial cells were cultured in 6 well plates and treated with $10^{-7} \mathrm{M} \mathrm{1,25}(\mathrm{OH}) 2$ D3 for $48 \mathrm{~h}$. After then pre-optimized concentrations of LPS or LTA was added to the wells for $8 \mathrm{~h}$. Based on the results of preliminary kinetic experiments, this time period was optimal for gene expression following endometrial cell stimulation with either LPS or LTA. After then, cells were harvested in RNA Bee and stored at $-70 \otimes \mathrm{C}$. 
Cytokine assay:

The concentrations of IL-6 (ebioscience, USA), IL-8 and TNF-a (BD biosciences, USA) were measured in cell culture supernatants by sandwich ELISA. The minimal detection limit for aforesaid cytokine assay sets were 7.5, 3.1, and $3.1 \mathrm{pg} / \mathrm{ml}$ for TNF-a, IL-6 and IL-8, respectively. All measurements were performed in triplicate.

Reverse transcriptase real time quantitative polymerase chain reaction (RT-qPCR):

Total RNA was extracted using commercial RNA extraction kit (Biosite-Sweden) according to manufacturer's protocol. The cDNA was synthesized for each sample using reverse transcriptase (Fermentas-Lithuania) based on the manufacturer instructions. The following primers were used for RTqPCR: TLR2 forward: 5'-ATTGTGCCCATTGCTCTTTC-3' Reverse: 5'-TTCTTCCTTGGAGAGGCTGA-3' [22]; TLR4 forward: 5'- GCTTCTTGCTGGCTGCATAA-3' reverse: GAAATGGAGGCACCCCTTC-3' [23]; MyD88 forward: 5'-GAGCGTTTCGATGCCTTCAT-3' reverse: 5'- CGGATCATCTCCTGCACAAA-3' [24] and $\beta$-actin was used as a housekeeping gene with following primers: Forward: 5'- AGCCTCGCCTTTGCCGA-3' reverse: 5'CTGGTGCCTGGGGCG-C3'[25]. To carry out qRT-PCR, SYBR Green (Takara) master mix was used. The amplification efficiency of target and reference genes were determined, then comparative $\Delta \triangle \mathrm{CT}$ method was used to analyze qRT-PCR data. Target genes expression were relatively compared and results were analyzed by Rest-rg software.

Western Blot:

TLR4 expression at the protein level was also studied using western blot according to the protocol published elsewhere [26]. In brief, cell lysate was prepared in a lysis buffer containing $\% 2$ SDS, 10\% glycerol, $50 \mathrm{mM}$ Dithiothreitol, and 0.1\% Bromophenol blue. Protein content of each cell lysate was measured using BCA assay. Proteins were resolved in a 10\% SDS-PAGE gel and bands were electrotransfered onto the PVDF membrane (Millipore, Bedford, USA). Immunobloting was performed using goat anti-human TLR4 (R\&D, USA) at $1 \mu \mathrm{g} / \mathrm{ml}$ for $2 \mathrm{~h}$ followed by washing and 45 min incubation with rabbit anti-goat Ig-HRP (Sinabiotech, Iran). Bands were visualized using ECL reagent (GE Healthcare, Sweden). Membranes were later stripped using Western Re-Probe (Calbiochem, SanDiego, CA, USA), reincubated for $2 \mathrm{hr}$ with agitation in polyclonal rabbit anti-beta actin antibody (Sigma, USA) as loading control followed by 45 min incubation in HRP-conjugated sheep anti-rabbit Ig (Sinabiotech) and processed as above. In negative control blots, primary antibody was substituted by equivalent dilutions of species-matched normal sera.

\section{Data Analysis}

Data were expressed as Scattor plot and mean. For comparison of different groups, either T-test or one way ANOVA analysis was used. The relative expression of target genes was normalized first with reference gene ( $\beta$-actin) and then with corresponding control. All data were analyzed and plotted by GraphPad Prism 6.07. 


\section{Results}

1,25 (OH)2 D3 downregulated LPS-induced production of pro-inflammatory cytokines by ESCs and WECs:

To assay the effect of 1,25 $(\mathrm{OH}) 2$ D3 on TNF-a, IL-6 and IL-8 production by ESCs, these cells were first primed with 1,25 $(\mathrm{OH}) 2$ D3 before stimulation with LPS. Concentration of cytokines was then measured in culture supernatants. LPS increased TNF-a production compared to non-treated control cells $(p<0.05)$. We observed that 1,25 (OH)2 D3 was able to significantly reduce TNF-a production by ESCs $(p<0.05)$ (Fig. 1a) comparing to the corresponding LPS-treated controls, and non-LPS treated control. Such treatment did not exert a considerable effect on IL-6 production by these cells (Fig. 1b), whereas LPS raised IL- 6 production by ESCs $(p<0.05)$.. In contrast to what observed in case of TNF-a, 1,25 $(\mathrm{OH}) 2 \mathrm{D} 3$ raised production of IL-8 at all LPS concentrations tested and non-LPS treated control as well $(p<0.01)$ (Fig. 1c). IL-8 was induced at all LPS concentrations comparing to non-treated control $(p<0.05) \ldots$

Based on our preliminary results in which LPS did not exert a significant impact on TNF-a production by WECs, effect of 1,25 $(\mathrm{OH}) 2$ D3 on this process was not studied. 1,25 $(\mathrm{OH}) 2 \mathrm{D} 3$ reduced IL-6 production by WECs which was already upregulated due to LPS treatment $(p<0.05)$ (Fig. 1d). LPS caused increased level of IL-8 production by WECs $(p<0.05)$. As with ESCs, 1,25 $(\mathrm{OH}) 2$ D3 upregulated LPS-induced production of IL-8 by WECs $(p<0.01)$ (Fig. 1e).

1,25 (OH)2 D3 downregulated LTA-induced production of pro-inflammatory cytokines by WECs:

The effect of 1,25 $(\mathrm{OH}) 2 \mathrm{D} 3$ on pro-inflammatory cytokines production by WECs following LTA stimulation was tested in parallel. Our data showed that 1,25 $(\mathrm{OH}) 2 \mathrm{D} 3$ reduced basal level $(p<0.05)$ and LTA-induced production of TNF-a $(p<0.01)$ (Fig. 2a) and IL-6 ( $p<0.05-0.01)$ (Fig. 2b), while increased IL8 production (Fig. 2 c) by LTA-treated $(p<0.05)$ and non-treated WECs $(p<0.01)$. Again, the effect of 1,25 $(\mathrm{OH}) 2 \mathrm{D} 3$ on unstimulated WECs mimicked its effect on LTA-activated cells.

\section{1,25 $(\mathrm{OH}) 2$ D3 reduced LPS-induced MyD88 expression in ESCc}

To study whether TLR4 and TLR2 signaling in WECs and ESCs following LPS and LTA stimulation is potentially affected by 1,25 $(\mathrm{OH}) 2$ D3 treatment, expression of TLR4, TLR2 and MyD88 transcript was investigated. Our results showed that 1,25 $(\mathrm{OH}) 2 \mathrm{D} 3$ neither affect expression of TLR4 by LPS-activated ESCs nor LTA-activated WECs (Fig. 3a and b). At the protein level, however, 1,25 (OH)2 D3 pre-treatment markedly reduced TLR4 protein expression by LPS-stimulated ESCs (Fig. 3c). Of note, LPS treatment of ESCs significantly induced MyD88 gene expression $(p<0.05)$. This effect was reversed when ESCs were pre-treated with 1,25 $(\mathrm{OH}) 2$ D3 before stimulation with LPS. 1,25 $(\mathrm{OH}) 2$ D3 itself had also an inhibitory effect on basal level of MyD88 expression in ESCs (Fig. 3d). In LTA-activated WECs, 1,25 (OH)2 D3 did not alter MyD88 gene expression (Fig. 3e).

\section{Discussion}


Bacterial and viral infections are considered a serious threat to gestation and developing embryo. Clinical studies have revealed a significant association between pregnancy complications and intrauterine infections. It was reported that infections account for etiology of more than $40 \%$ of preterm birth cases [27] and in about $80 \%$ of preterm births before 30 weeks of gestation the footprint of infections can be tracked. Infections have also been shown to be linked with spontaneous abortion and preeclampsia. The pathogenesis behind infection-induced pregnancy complications comprises inflammation and proinflammatory cytokines production by PAMPs-activated local immune cells [28].

Endometrium is a site in which a precise tuning of inflammatory responses in necessary for successful pregnancy. It expresses a full range of TLR molecules [29] pointing to the importance of local immune defense against a wide spectrum of microorganisms. We also previously showed that cultured human endometrial cells express TLR2, TLR4 and MyD88 [20]. Although TLRs are essential for female reproductive tract (FRT) defense against invading pathogens, the lack of TLR negative regulatory system might be associated with inflammatory responses leading to harmful consequences. 1,25 (OH)2 D3 is a part of such regulatory network known to be effective in the downregulation of pro-inflammatory cytokine production [29,30], induction of regulatory $T$ cells and inhibition of antigen presentation [30]. Notably, component of vitamin D3 functional network is active in male and female reproductive tract[14, 16, 31], [15]. We showed that human ESCs express vitamin D receptor and 1a hydroxylase, the enzyme responsible for the production of active form of vitamin D3, implying that these cells are the target of vitamin D3 action. Notably, these cells are also produce active form of vitamin D3 pointing to the central role of this hormone in FRT [15].

Here, we unrevealed an unprecedented action of vitamin D3 in FRT. We showed that 1,25 (OH)2 D3 significantly counteract with LPS-induced TNF-a production by ESCs and IL- 6 production by WECs. Similarly, 1,25 $(\mathrm{OH}) 2$ D3 reduced TNF-a and IL-6 production by LTA-stimulated WECs. Anti-inflammatory action of 1,25(OH)2D3 has been reported earlier showing that this hormone prevents production of inflammatory cytokines by monocytes [32]. It has also been reported that 1,25(OH)2D halts IL-6, TNF-a and GM-CSF-2 expression in primary culture of human decidua and syncytiotrophoblast [33-35]. Diaz et al. demonstrated that in cultured human trophoblasts calciferol reduces TNF-a gene expression. They also gave evidence that calciferol influence Th1 cytokines production through regulating TNF-a function in these cells. These findings imply that there is genomic mechanisms governing anti-inflammatory effects of calciferol. There are several genes such as; TNF- $a$, IL- 6 and IFN- $\gamma$ which that are recognized by responsive elements to 1,25 $(\mathrm{OH}) 2 \mathrm{D} 3$ [34]. Besides, vitamin D is a part of FRT immune network which exerts an essential role in anti-bacterial defense through augmenting macrophage function and antibacterial agent, cathelicidin [36].

Despite of inhibitory effect of 1,25 (OH)2 D3 on IL-6 and TNF-a production, we observed that, 1,25 (OH)2 D3 increased IL-8 production in WECs and ESCs. IL-8 has chemotactic effects on neutrophils in both in vivo and in vitro and involved in hemostasis of innate immune system in endometrium [37]. Besides, it is a potential growth factor for endometrial stromal cell [38]. In this regard, it can be concluded that 1,25 
$(\mathrm{OH}) 2 \mathrm{D} 3$ have a dual role in controlling inflammatory responses of endometrium and at the same time potentiate innate defense mechanisms of endometrium.

Next, we showed that LPS-induced upregulation of MyD88 gene expression in ESCs was normalized when these cells were pre-treated with 1,25 $(\mathrm{OH}) 2 \mathrm{D} 3$. This effect may explain in part aforementioned anti-inflammatory action of 1,25 (OH)2 D3 on ESCs. MyD88 acts as the common adaptor protein for TLR2 and TLR4 and downregulation of MyD88 expression could expectedly inhibits TLR2 and TLR4 signaling leading to diminished levels of pro-inflammatory cytokines production. Moreover, we found that 1,25 $(\mathrm{OH}) 2$ D3 pre-treatment markedly reduced LPS-induced TLR-4 protein expression by ESCs. This effect could synergistically act with MyD88 downregulation and strongly inhibit LPS-induced inflammatory responses. In line with our findings, Sadeghi et al. demonstrated that 1,25 (OH)2D3 amplify anti-microbial functions in myeloid cells whereas it decreases TLR2 and TLR4 expression in monocytes in which it creates a condition less sensitive to PAMPs [39]. Indeed, Do et al. ascribed high expression of TLR on monocytes to low 1,25(OH)2D3 serum level in patients with Behcet's disease [40]. Despite our finding at the protein level, we did not observe negative regulatory effect of 1,25 (OH)2D3 on TLR2 and TLR4 gene expression which could be attributed to the mRNA instability. Indeed, 1,25 (OH)2 D3 only succeeded to dampen MyD88 expression in ESCs stimulated with LPS but failed to do the same in WECs stimulated with LTA. TLR4 is MYD88 dependent [20], while this adaptor protein is not critical for TLR2 signaling [41]. We also demonstrated that LTA could not cause a raise of MyD88 gene expression in WECs [20]. In this context, downregulation of pro-inflammatory cytokines in LTA-treated WECs following $1,25(\mathrm{OH}) 2 \mathrm{D} 3$ treatment might be due to the effect of this hormone on collateral signaling adaptor proteins.

Taken all these, it can be inferred that 1,25 $(\mathrm{OH}) 2 \mathrm{D} 3$ is an immunomodulatory molecule essential for maintenance of endometrial immune homeostasis through controlling potentially harmful inflammatory responses and brushing off unwanted immune reactions associated with FRT infections. In line with this conclusion, recent findings also linked vitamin D deficiency to increased risk of FRT infections and production of pro-inflammatory cytokines [42]. Further clinical studies are needed to explore full spectrum of other immunomodulatory facets of 1,25 $(\mathrm{OH}) 2 \mathrm{D} 3$ in female reproductive tract.

\section{Abbreviations}

ESCs:Endometrial stromal cells, LPS:Lipopolysaccharide, WECs:Whole endometrial cells, LTA:Lipoteichoic acid, TLRs:Toll like receptors, PAMPs:Pathogen-associated molecular patterns, FRT:Female reproductive tract

\section{Declarations}

Acknowledgment: This study was supported by grants from Avicenna Research Institute (870106-019) and Tehran University of Medical Sciences (87-03-30-7757). 
Conflict of interest: The authors declare no conflict of interest.

\section{References}

1. Huleihel M, Golan H, Hallak MJRB, Endocrinology: Intrauterine infection/inflammation during pregnancy and offspring brain damages: possible mechanisms involved. Reproductive Biology and Endocrinology 2004, 2(1):17.

2. Hirata T, Osuga $Y$, Hamasaki K, Hirota $Y$, Nose E, Morimoto $C$, Harada $M$, Takemura $Y$, Koga $K$, Yoshino 0 et al: Expression of toll-like receptors 2, 3, 4, and 9 genes in the human endometrium during the menstrual cycle. Journal of reproductive immunology 2007, 74(1-2):53-60.

3. Takeda K, Kaisho T, Akira S: TOLL-LIKE RECEPTORS. Annual Review of Immunology 2003, 21(1):335-376.

4. Sato S, Kiyono HJCoiv: The mucosal immune system of the respiratory tract. 2012, 2(3):225-232.

5. Hirata T, Osuga Y, Hamasaki K, Hirota Y, Nose E, Morimoto C, Harada M, Takemura Y, Koga K, Yoshino 0 : Expression of toll-like receptors 2,3,4, and 9 genes in the human endometrium during the menstrual cycle. Journal of reproductive immunology 2007, 74(1-2):53-60.

6. Aflatoonian R, Tuckerman E, Elliott S, Bruce C, Aflatoonian A, Li T, Fazeli A: Menstrual cycledependent changes of Toll-like receptors in endometrium. Human Reproduction 2006, 22(2):586-593.

7. Fazeli A, Bruce $C$, Anumba DO: Characterization of Toll-like receptors in the female reproductive tract in humans. Human reproduction (Oxford, England) 2005, 20(5):1372-1378.

8. Medzhitov R: Toll-like receptors and innate immunity. Nat Rev Immunol 2001, 1(2):135-145.

9. Abrahams VM, Bole-Aldo P, Kim YM, Straszewski-Chavez SL, Chaiworapongsa T, Romero R, Mor G: Divergent trophoblast responses to bacterial products mediated by TLRs. J Immunol 2004, 173(7):4286-4296.

10. Chaouat G, Menu E, Clark DA, Dy M, Minkowski M, Wegmann TG: Control of fetal survival in CBA $\mathbf{x}$ DBA/2 mice by lymphokine therapy. Reproduction 1990, 89(2):447-458.

11. Paradisi R, Porcu E, Venturoli S, Maldini-Casadei M, Boni PJAJoRI: Maternal serum levels of proinflammatory cytokines in missed and threatened abortion. 2003, 50(4):302-308.

12. Makhseed M, Raghupathy R, El-Shazly S, Azizieh F, Al-Harmi JA, Al-Azemi MMK: Pro-inflammatory Maternal Cytokine Profile in Preterm Delivery. American Journal of Reproductive Immunology 2003, 49(5):308-318.

13. Mathieu $C$, Adorini $L$ : The coming of age of 1,25-dihydroxyvitamin D3 analogs as immunomodulatory agents. Trends in Molecular Medicine 2002, 8(4):174-179.

14. Mahmoudi AR, Zarnani AH, Jeddi-Tehrani M, Katouzian L, Tavakoli M, Soltanghoraei $H$, Mirzadegan $E$ : Distribution of vitamin $D$ receptor and 1a-hydroxylase in male mouse reproductive tract. Reproductive Sciences 2013, 20(4):426-436.

15. Tavakoli M, Jeddi-Tehrani M, Salek-Moghaddam A, Rajaei S, Mohammadzadeh A, Sheikhhasani S, Kazemi-Sefat G-E, Zarnani AH: Effects of 1, $25(\mathrm{OH}) 2$ vitamin D3 on cytokine production by 
endometrial cells of women with recurrent spontaneous abortion. Fertility and sterility 2011, 96(3):751-757.

16. Rajaei S, Mirahmadian M, Jeddi-Tehrani M, Tavakoli M, Zonoobi M, Dabbagh A, Zarnani AH: Effect of $1,25(\mathrm{OH}) 2$ vitamin D3 on cytokine production by endometrial cells of women with repeated implantation failure. Gynecological Endocrinology 2012, 28(11):906-911.

17. Etten Ev, Mathieu C: Immunoregulation by 1,25-dihydroxyvitamin D3: Basic concepts. The Journal of Steroid Biochemistry and Molecular Biology 2005, 97(1-2):93-101.

18. Zehnder D, Evans KN, Kilby MD, Bulmer JN, Innes BA, Stewart PM, Hewison M: The Ontogeny of 25Hydroxyvitamin D3 1[alpha]-Hydroxylase Expression in Human Placenta and Decidua. The American Journal of Pathology 2002, 161(1):105-114.

19. Sadeghi K, Wessner B, Laggner U, Ploder M, Tamandl D, Friedl J, Zugel U, Steinmeyer A, Pollak A, Roth $\mathrm{E}$ et al: Vitamin D3 down-regulates monocyte TLR expression and triggers hyporesponsiveness to pathogen-associated molecular patterns. European journal of immunology 2006, 36(2):361-370.

20. Rashidi N, Mirahmadian M, Jeddi-Tehrani M, Rezania S, Ghasemi J, Kazemnejad S, Mirzadegan E, Vafaei S, Kashanian M, Rasoulzadeh Z: Lipopolysaccharide-and lipoteichoic acid-mediated proinflammatory cytokine production and modulation of TLR2, TLR4 and MyD88 expression in human endometrial cells. Journal of reproduction \& infertility 2015, 16(2):72.

21. Delbandi AA, Mahmoudi M, Shervin A, Zarnani AH: 1, 25-Dihydroxy Vitamin D3 Modulates Endometriosis-Related Features of Human Endometriotic Stromal Cells. American Journal of Reproductive Immunology 2016, 75(4):461-473.

22. Smith MF, Mitchell A, Li G, Ding S, Fitzmaurice AM, Ryan K, Crowe S, Goldberg JB: Toll-like receptor (TLR) 2 and TLR5, but not TLR4, are required for Helicobacter pylori-induced NF-KB activation and chemokine expression by epithelial cells. Journal of Biological Chemistry 2003, 278(35):3255232560 .

23. Abreu MT, Arnold ET, Thomas LS, Gonsky R, Zhou Y, Hu B, Arditi M: TLR4 and MD-2 expression is regulated by immune-mediated signals in human intestinal epithelial cells. Journal of Biological Chemistry 2002, 277(23):20431-20437.

24. Zarember KA, Godowski PJ: Tissue expression of human Toll-like receptors and differential regulation of Toll-like receptor mRNAs in leukocytes in response to microbes, their products, and cytokines. The journal of immunology 2002, 168(2):554-561.

25. Radonic A, Thulke S, Mackay IM, Landt O, Siegert W, Nitsche A: Guideline to reference gene selection for quantitative real-time PCR. Biochemical and biophysical research communications 2004, 313(4):856-862.

26. Shahbazi M, Jeddi-Tehrani M, Zareie M, Salek-Moghaddam A, Akhondi M, Bahmanpoor M, Sadeghi $M, Z a r n a n i$ : Expression profiling of vitamin $D$ receptor in placenta, decidua and ovary of pregnant mice. Placenta 2011, 32(9):657-664.

27. Mendz GL, Kaakoush NO, Quinlivan JA: Bacterial aetiological agents of intra-amniotic infections and preterm birth in pregnant women. Frontiers in cellular and infection microbiology 2013, 3:58. 
28. Krishnan L, Guilbert LJ, Wegmann TG, Belosevic M, Mosmann TRJTJol: T helper 1 response against Leishmania major in pregnant C57BL/6 mice increases implantation failure and fetal resorptions. Correlation with increased IFN-gamma and TNF and reduced IL-10 production by placental cells. 1996, 156(2):653-662.

29. Aflatoonian R, Tuckerman E, Elliott SL, Bruce C, Aflatoonian A, Li TC, Fazeli A: Menstrual cycledependent changes of Toll-like receptors in endometrium. Human reproduction (Oxford, England) 2007, 22(2):586-593.

30. Bartels LE, Hvas CL, Agnholt J, Dahlerup JF, Agger R: Human dendritic cell antigen presentation and chemotaxis are inhibited by intrinsic 25-hydroxy vitamin D activation. International immunopharmacology 2010, 10(8):922-928.

31. Zarnani AH, Shahbazi M, Salek-Moghaddam A, Zareie M, Tavakoli M, Ghasemi J, Rezania S, Moravej A, Torkabadi E, Rabbani H: Vitamin D3 receptor is expressed in the endometrium of cycling mice throughout the estrous cycle. Fertility and sterility 2010, 93(8):2738-2743.

32. Giulietti A, van Etten E, Overbergh L, Stoffels K, Bouillon R, Mathieu C: Monocytes from type 2 diabetic patients have a pro-inflammatory profile. 1,25-Dihydroxyvitamin $D(3)$ works as antiinflammatory. Diabetes research and clinical practice 2007, 77(1):47-57.

33. Evans KN, Nguyen L, Chan J, Innes BA, Bulmer JN, Kilby MD, Hewison M: Effects of 25Hydroxyvitamin D3 and 1,25-Dihydroxyvitamin D3 on Cytokine Production by Human Decidual Cells. Biology of Reproduction 2006, 75(6):816-822.

34. Diaz L, Noyola-Martinez N, Barrera D, Hernandez G, Avila E, Halhali A, Larrea F: Calcitriol inhibits TNFalpha-induced inflammatory cytokines in human trophoblasts. Journal of reproductive immunology 2009, 81(1):17-24.

35. Liu N, Kaplan AT, Low J, Nguyen L, Liu GY, Equils O, Hewison M: Vitamin D Induces Innate Antibacterial Responses in Human Trophoblasts via an Intracrine Pathway. Biology of Reproduction 2009, 80(3):398-406.

36. Martineau AR, Wilkinson KA, Newton SM, Floto RA, Norman AW, Skolimowska K, Davidson RN, Sørensen OE, Kampmann B, Griffiths CJ: IFN-y-and TNF-independent vitamin D-inducible human suppression of mycobacteria: the role of cathelicidin LL-37. The Journal of Immunology 2007, 178(11):7190-7198.

37. Shen L, Fahey JV, Hussey SB, Asin SN, Wira CR, Fanger MW: Synergy between IL-8 and GM-CSF in reproductive tract epithelial cell secretions promotes enhanced neutrophil chemotaxis. Cellular immunology 2004, 230(1):23-32.

38. Arici A, Seli E, Zeyneloglu HB, Senturk LM, Oral E, Olive DL: Interleukin-8 induces proliferation of endometrial stromal cells: a potential autocrine growth factor. The Journal of Clinical Endocrinology \& Metabolism 1998, 83(4):1201-1205.

39. Sadeghi K, Wessner B, Laggner U, Ploder M, Tamandl D, Friedl J, Zügel U, Steinmeyer A, Pollak A, Roth E: Vitamin D3 down-regulates monocyte TLR expression and triggers hyporesponsiveness to pathogen-associated molecular patterns. European journal of immunology 2006, 36(2):361-370. 
40. Do JE, Kwon SY, Park S, Lee ES: Effects of vitamin D on expression of Toll-like receptors of monocytes from patients with Behcet's disease. Rheumatology (Oxford, England) 2008, 47(6):840848.

41. Kenny EF, Talbot S, Gong M, Golenbock DT, Bryant CE, O'Neill LA: MyD88 adaptor-like is not essential for TLR2 signaling and inhibits signaling by TLR3. The Journal of Immunology 2009:jimmunol. 0901140.

42. Akoh CC, Pressman EK, Cooper E, Queenan RA, Pillittere J, O'Brien KO: Low vitamin D is associated with infections and proinflammatory cytokines during pregnancy. Reproductive Sciences 2018, 25(3):414-423.

\section{Figures}


a

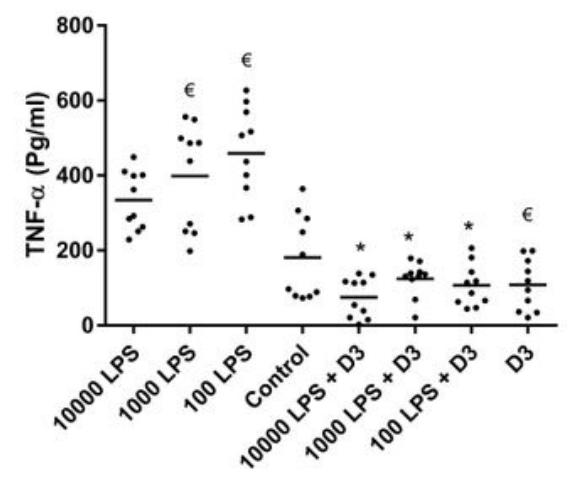

C

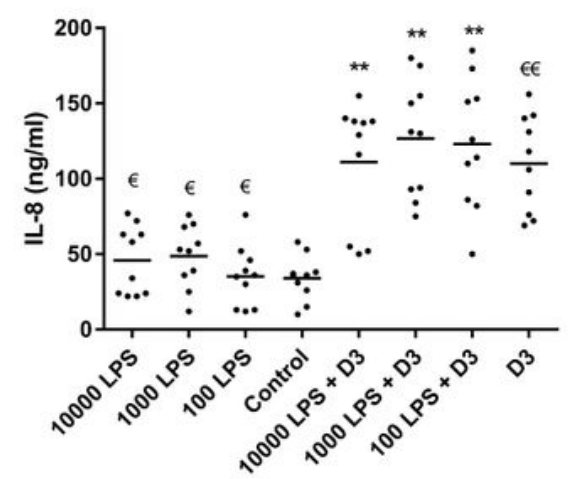

e

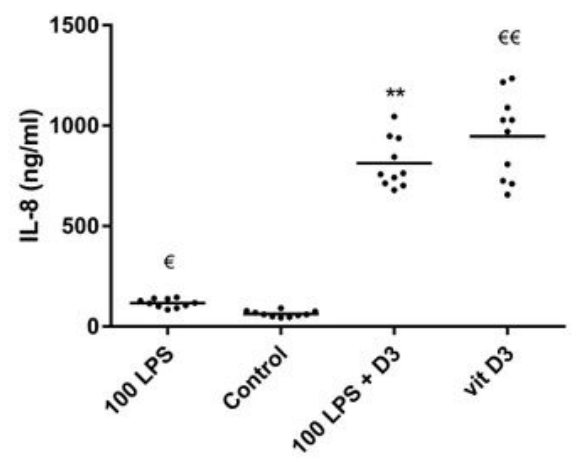

b

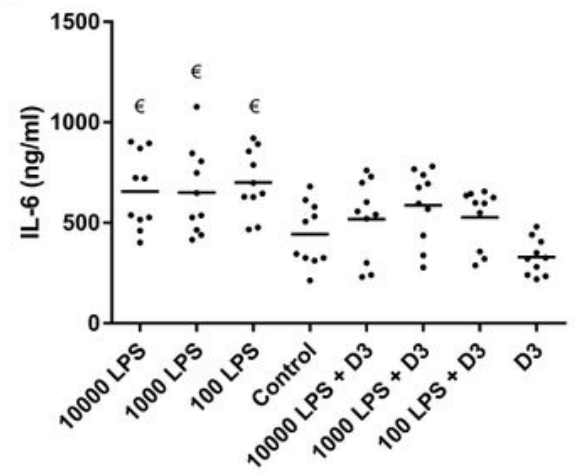

d

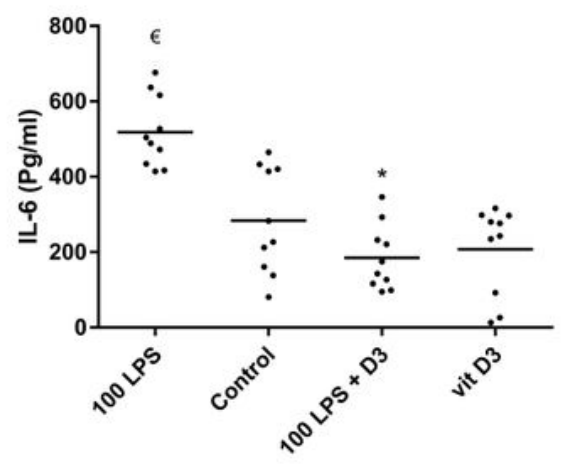

\section{Figure 1}

Effect of 1,25 (OH)2 D3 on pro-inflammatory cytokine production by LPS-activated ESCs and WECs. Endometrial stromal cells (ESCs) (a-c) and whole endometrial cells (WECs) (d and e) were pre-treated with 1,25 (OH)2 D3 before stimulation with LPS (100-10000 ng/ml) and levels of TNF- $a(a), ~ I L-6(b$ and d) and IL-8 (c and e) were measured in culture supernatants. $(n=10), *, €: p<0.05$. **, $€ €: p<0.01$. $€$ : compared to control and *: compared to corresponding LPS-stimulated cells without 1,25 (OH)2 D3 pre-treatment. 
a
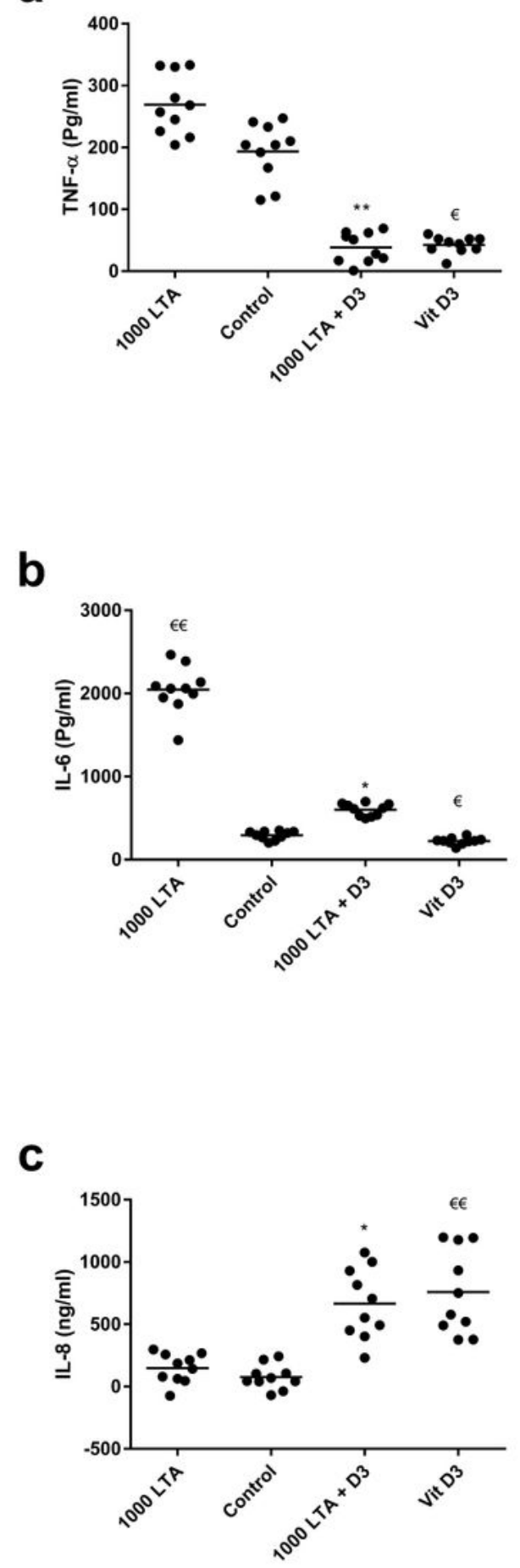

\section{Figure 2}

Effect of 1,25 $(\mathrm{OH}) 2$ D3 on pro-inflammatory cytokine production by LTA-activated WECs. Whole endometrial cells (WECs) were pre-treated with 1,25 (OH)2 D3 before stimulation with LTA $(1000 \mathrm{ng} / \mathrm{ml})$ and levels of TNF-a (a), IL-6 (b) and IL-8 (c) were measured in culture supernatants. $(n=10), *, €: p<0.05 . * *$, $€ €: p<0.01$. $€$ : compared to control and *: compared to corresponding LPS-stimulated cells without 1,25 $(\mathrm{OH}) 2 \mathrm{D} 3$ pre-treatment. 

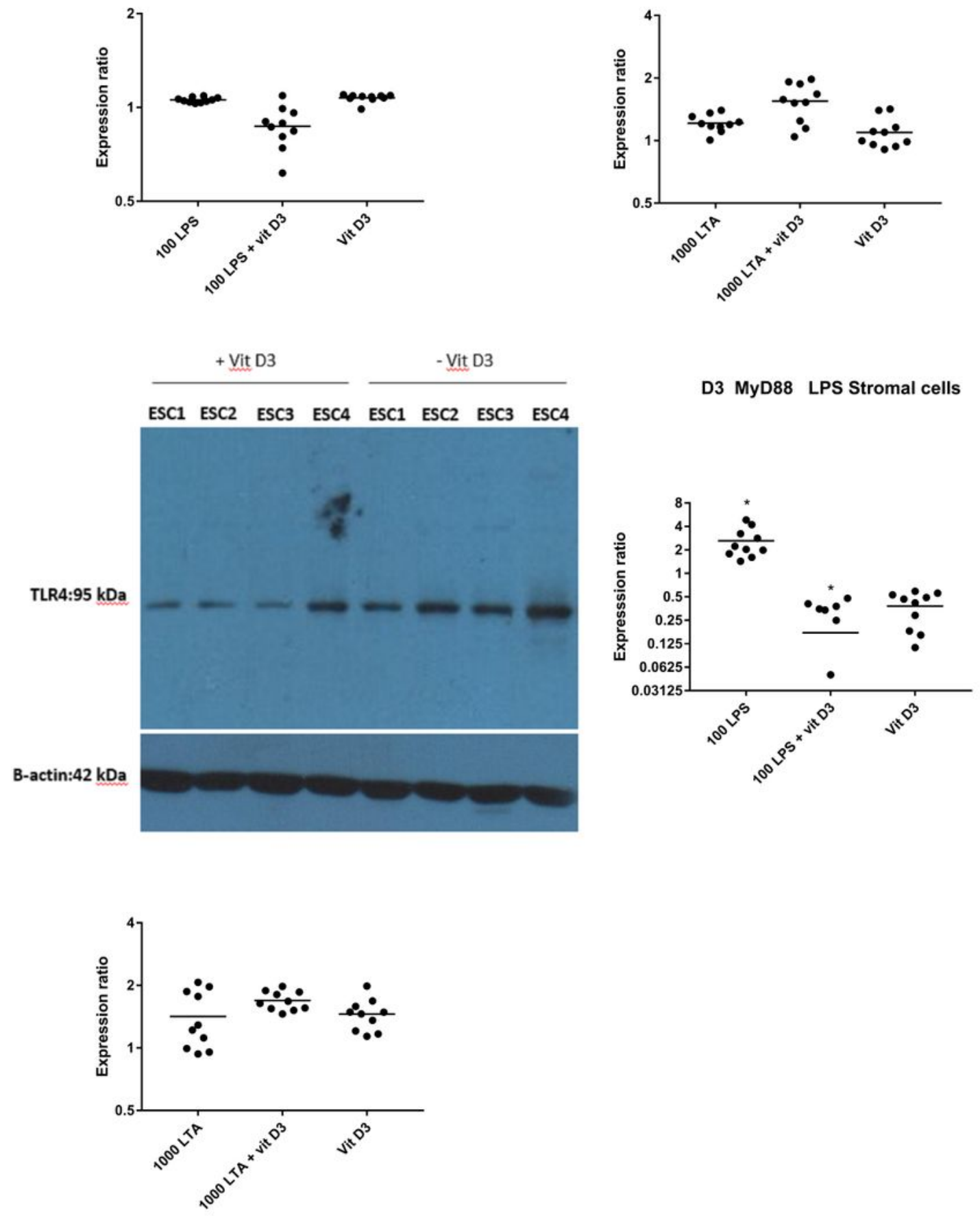

Figure 3

Effect of 1,25 (OH)2 D3 on TLR2, TLR4 and MyD88 expression in ESCs and WECs. Endometrial stromal cells (ESCs) ( $a$ and d) and whole endometrial cells (WECs) (b and e) were pre-treated with 1,25 (OH)2 D3 before stimulation with LPS or LTA and the expression of TLR4 (a), TLR2 (b) and MyD88 ( $d$ and e) transcripts were quantified by real time PCR. Expression of TLR4 protein was also investigated in ESCs 
after pre-treatment with 1,25 (OH)2 D3 and stimulation with LPS (c). Relative expression was calculated in each group in reference to corresponding control. $(n=10), *: p<0.05$. 